\title{
Objective Quality Assessment Measurement for Typhoon Cloud Image Enhancement
}

\author{
Changjiang Zhang, Juan Lu, and Jinshan Wang \\ College of Mathematics, Physics and Information Engineering, \\ Zhejiang Normal University, Jinhua, China \\ zcj74922@zjnu.edu.cn
}

\begin{abstract}
There are kinds of enhancement methods for satellite image, however, visual quality of them are basically assessed by human eyes. This can result in wrong identification. This will result in wrong prediction for center and intensity of the typhoon. It is necessary to find an objective measure to evaluate the visual quality for enhanced typhoon cloud image. In order to solve this problem, we give an objective assessment measurement based on information, contrast and peak-signal-noise-ratio. We design an experiment to certify the proposed measure by using the typhoon cloud images which are provided by China Meteorological Administration, China National Satellite Meteorological Center.
\end{abstract}

Keywords: Assessment; typhoon; satellite image; enhancement.

\section{Introduction}

There are many kinds of noise in a typhoon cloud image. If they cannot be efficiently reduced, they may affect the overall image quality to the extent that it is impossible to extract some important information. For example, the noise may disturb the procedure of locating the center position, when we try to predict the moving path of the typhoon. In addition, the contrast of some typhoon cloud images may be poor, which may affect to accurately segment the helical cloud band from the typhoon cloud image. Therefore, it is important to efficiently reduce the noise and enhance the contrast in a typhoon cloud image.

Recently, much good work has been carried out in satellite cloud image enhancement. Ref. [1] developed an approach by merging the data from several satellite images of the same area in order to improve the spatial resolution of the sensor. This enhancement technique is called Data Cumulation. The paper starts with the theory of sampling image data over a scene, discusses the theoretical background of the approach and describes it implementation. Simulated Data Cumulation has been carried out using both artificial targets and satellite image data as well. Ref. [2] pointed out that threshold selection is an important problem for the purpose of image enhancement. Image enhancement consists of subdividing the intensity gray levels into bands, such that the resulting image presents more contrast, and less gray levels. One means of achieving threshold selection is through the use of gray tone spatial dependency 
matrices. A function of gray levels is computed from a co-occurence matrix of the image and then a threshold is chosen which corresponds to the etrema of this function. This article describes the algorithm of GTRLM and the results of the application of this technique to some satellite images. Ref. [3] presented a method for the improvement of the visual quality of satellite and aerial images. It helps geographers in their visual interpretation of urban growth in developing countries. This method is based on local contrast estimation. Results of the application of the algorithms to aerial and satellite images are discussed. Ref. [4] accomplished satellite image enhancement and smoothing towards automatic feature extraction through an effective serial application of anisotropic diffusion processing and alternating sequential filtering. A robust anisotropic diffusion filtering is used with Tukey's biweight robust error norm for "edgestopping" function. A well-known class of morphological filters, alternating sequential filtering is applied afterwards for a more extended enhancement and smoothing.

Recently, the wavelet transform has been widely applied to the image enhancement. Some image enhancement algorithms only consider the enhancement and do not care about noise reduction. For example, Fu, J.C. and Wan, Y. used the improved histogram equalization in wavelet domain to enhance the image Ref. [5]. Temizel, A. proposed two algorithms to enhance an image resolution by the estimation of detail wavelet coefficients at high resolution scales and cycle-spinning methodology in the wavelet domain respectively [6]. Xiao, D. respectively presented an algorithm to enhance the contrast of an image by modifying both coarse and detail coefficients[7]. Heric, D. introduced a novel image enhancement technique based on the multiscale singularity detection with an adaptive threshold whose value is calculated via the maximum entropy measure in the directional wavelet domain [8]. Some image enhancement algorithms only consider noise reduction and do not care about detail enhancement. For example, Ercelebi, E. proposed a method by applying lifting-based wavelet domain Wiener filter to enhance the contrast of an image [9]. The proposed method transforms an image into the wavelet domain using lifting-based wavelet filters and then applies a Wiener filter in the wavelet domain and finally transforms the result into the spatial domain. Many enhancement algorithms consider both detail enhancement and noise reduction. For example, Pengxin Zeng proposed a waveletbased algorithm for image contrast enhancement [10]. The approach treats the correlation between wavelet planes as an indication of the likelihood that noise is present. Then, it modifies the wavelet transform coefficients at different scales in different degrees by a pointwise nonlinear transformation. The algorithm achieves an excellent balance between the enhancement of subtle image detail, and the avoidance of noise amplification. Luo, Gaoyong presented a new method of x-ray image denoising based on fast lifting wavelet thresholding for general noise reduction and spatial filtering for further denoising by using a derivative model to preserve edges [11]. Belousov, Artem A. described a developed two-phase full-color image enhancement algorithm [12]. During the first phase the picture is denoised based wavelet thresholding. During the second phase brightness and contrast are automatically tuned up using evolutionary algorithm.

Although many above methods have been proposed to enhance image, however, most of methods assess the image quality by subjective measure. This will result in different assessment for different men. It is necessary to develop an efficient objective 
assessment measure for an image. Especially, for typhoon cloud image, this is very necessary because this will affect the center location of the typhoon and main body segmentation of cloud series of the typhoon. Further, this will result in an incorrect prediction for moving path or intensity of the typhoon.

\section{Objective Assessment Measure for Typhoon Cloud Image}

Digital images are subject to a wide variety of distortions during acquisition, processing, compression, storage, transmission and reproduction, any of which may result in a degradation of visual quality. For applications in which images are ultimately to be viewed by human beings, the only "correct" method of qualifying visual image quality is through subjective evaluation. In practice, however, subjective evaluation is usually too inconvenient, time-consuming and expensive [13]. The goal of research in objective image quality assessment is to develop quantitative measures that can automatically predict perceived image quality.

An objective image quality metric can play a variety of roles in image processing applications. First, it can be used to dynamically monitor and adjust image quality. Second, it can be used to optimize algorithms and parameter settings of image processing systems. Third, it can be used to benchmark image processing systems and algorithms. We construct a quality measure from the perspective of image information in order to assess the enhanced image quality. The system separates the task of enhanced image quality measurement into three comparisons: information entropy, contrast and signal-noise-ratio. First, the information entropy of each enhanced image is compared. The better the enhanced image quality is, the bigger the information entropy is. Second, the contrast of each enhanced image is compared. Assuming a discrete signal, the mean intensity can be written as

$$
\mu_{x}=\frac{1}{N} \sum_{i=1}^{N} x_{i}
$$

We remove the mean intensity from the signal. In discrete form, the resulting signal $\mathrm{x}-\mu_{x}$ corresponds to the projection of vector $\mathrm{x}$ onto the hyperplane defined by

$$
\sum_{i=1}^{N} x_{i}=0
$$

We used the standard deviation (the square root of variance) as an estimate of the signal contrast. An unbiased estimate in discrete form is given by

$$
\mathrm{c}=\left(\frac{1}{N-1} \sum_{i=1}^{N}\left(x_{i}-\mu_{x}\right)^{2}\right)^{\frac{1}{2}}
$$

The better the enhanced image quality is, the bigger $c$ is.

Third, the peak-signal-noise-ratio (PSNR) of enhanced image is compared. 


$$
\mathrm{p}=10 \cdot \log \left(\frac{M N \cdot \max \left(F_{i j}{ }^{2}\right)}{\sum_{i=1}^{M} \sum_{j=1}^{N}\left(F_{i j}-G_{i j}\right)^{2}}\right)
$$

Where $F_{i j}$ and $G_{i j}$ are gray level value at $(i, j)$ in original image and enhanced image respectively. $M$ and $N$ are width and height of the original image respectively. The better the enhanced image quality is, the bigger $\mathrm{p}$ is. Finally, the three components are combined to yield an overall measure

$$
S=f(e, c, p)
$$

Generally, the better the enhanced image quality is, the bigger $\mathrm{p}$ is. However, the PSNR may become small after an image is enhanced and reduced noise although the overall quality of an image is good. Therefore we construct following overall measure to assess the quality of an enhanced image:

$$
\mathrm{S}=\mathrm{e}^{\alpha} \cdot \mathrm{c}^{\beta} \cdot\left[\operatorname{sign}(\mathrm{p}) \cdot|\mathrm{p}|^{\gamma}\right]
$$

where $\alpha>0, \beta>0$ and $\gamma>0$ are parameters used to adjust the relative importance of the three components. In order to simplify the expression and above analysis to PSNR, we set $\alpha=\beta=1$ and $\gamma=\frac{1}{5}$ in this paper. This results in a specific form of the following index

$$
\mathrm{S}=\mathrm{e} \cdot \mathrm{c} \cdot\left[\operatorname{sign}(\mathrm{p}) \cdot|\mathrm{p}|^{\frac{1}{5}}\right]
$$

The better the enhanced image quality is, the bigger $\mathrm{S}$ is.

\section{Experimental Results}

In experiments, nine infrared typhoon cloud images, which are provided by China Meteorological Administration, China National Satellite Meteorological Center, are used to verify the efficiency of proposed algorithm, where three typhoon cloud images are corrupted by additive gauss white noise (GWN). We will compare the performance between the proposed algorithm (ZCJ) [14], histogram equalization (HE), AFL method [15], WYQ method [16], XLZ method [17] and un-sharpened mask method (USM). Fig.1 (a)-(b) respectively represent original typhoon cloud image (typhoon No. 0425) and the corresponding noisy one (peak-signal-noise-ratio is 10dB). Fig.1 (c)-(h) respectively shows denoising and enhancement results by HE, AFL, WYQ, XLZ, USM and ZCJ. 


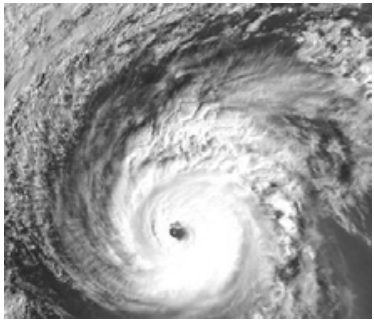

(a) Typhoon (No. 0425) cloud image

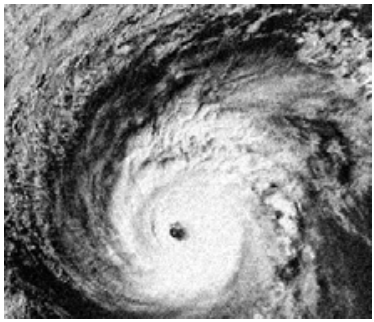

(c) HE

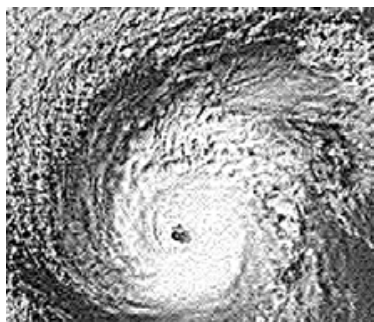

(e) WYQ

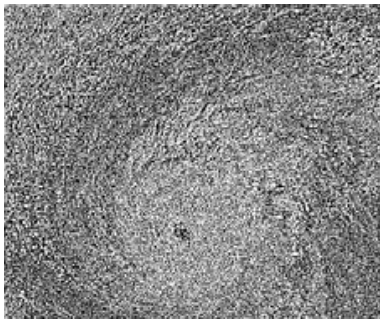

(g) USM

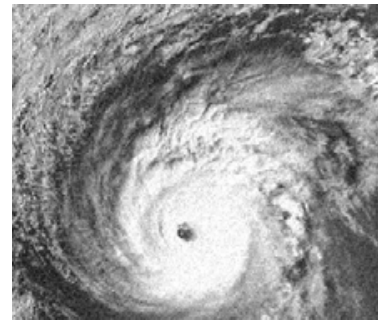

(b) Noisy image $(\mathrm{PSNR}=10 \mathrm{~dB})$

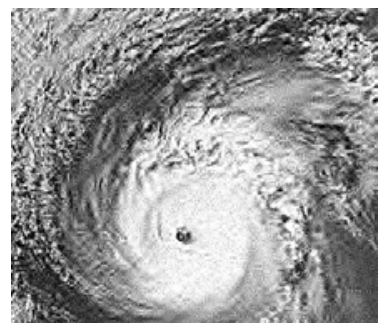

(d) AFL

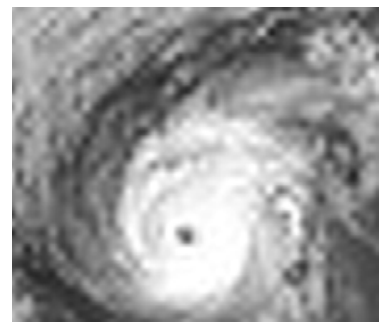

(f) XLZ

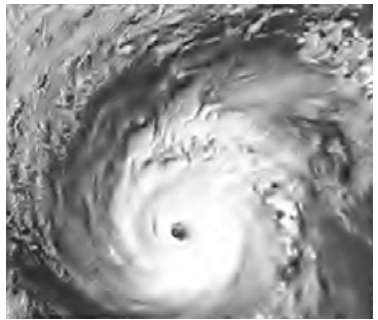

(h) ZCJ

Fig. 1. Typhoon (No. 0425- NOCK-TEN) cloud image enhancement results

According to Fig.1, we can see that HE method, AFL method and USM only considers enhancing contrast of an image while noise is also enhanced while enhancing the contrast of the typhoon cloud image. AFL method, which is proposed based on the 
classical discrete wavelet transform, has a better result than HE method and USM method, however, there are some undesired spot in the enhanced typhoon cloud image. Both WYQ method and XLZ method consider noise suppression while enhancing the contrast of the typhoon cloud image. WYQ method has enlarged the background bur while enhancing contrast of the typhoon cloud image. XLZ method has a good denoising result. However, the details in the typhoon cloud image are blurred. Noise in the enhanced image by USM method is greatly enlarged. The eye and cloud bands in the cloud image are emerged into the background. Compared with five other methods, ZCJ method is the best in visual quality. Both contrast and details are good, this is obvious in Fig.1 (h). Noise in Fig.1 (b) is also reduced well and eye region and main cloud bands of the typhoon are well kept.

In order to objectively assess the enhanced images quality, Equation (7) is used to accomplish the work. Table 1 lists the scores of enhanced images with different peaksignal-noise-ratio (PSNR). According to Table 1, we can see that the scores of enhanced images are becoming lower and lower with the increasing of standard deviation of noise. Compared with five other methods, the scores of enhanced images using the proposed algorithm are the highest. This exactly proves the advantage of the proposed algorithm. In order to certify the performance of the proposed assessment measure, we give thirty other typhoon cloud images to test the performance of the proposed measurement. We respectively select ten typhoon cloud images in 2004 (0401- SUDAL, 0402-NIDA, 0404-CONSON, 0406-DIANMU, 0409-KOMPASU, 0413- MERANTI, 0414-RANANIM，0418-AERE，0422-MEARI, and 0423-MA-ON), 2005 (0502ROKE, 0503-SONCA, 0504-NESAT, 0505-HAITANG, 0509-MATSA, 0511MAWAR, 0514-NABI, 0516-VICENTE, 0518-DAMREY, and 0522-TEMBIN) and 2006 (0601- CHANCHU, 0603-EWINIAR, 0606-PRAPIROON, 0611-SONAMU, 0613- SHANSHAN, 0616-BEBINCA，0617-RUMBIA，0619-CIMARON，0621DURIAN, and 0623-Trami) to test the performance of the proposed assessment measure. The meaning of "0401- SUDAL" can be explained as follows: name of typhoon is SUDAL, which is the first typhoon in 2004. Similar other spellings have the similar meanings.

Fig.2-Fig.4 show the test typhoon cloud images from 2004 to 2006. The five cloud images of the first row in Fig.2 are respectively 0401- SUDAL, 0402-NIDA, 0404-CONSON, 0406-DIANMU, and 0409-KOMPASU. The five cloud images of the second row in Fig.2 respectively show 0413- MERANTI, 0414-RANANIM, 0418-AERE, 0422-MEARI, and 0423-MA-ON. The five cloud images of the first row in Fig.3 are respectively 0502-ROKE, 0503-SONCA, 0504-NESAT, 0505HAITANG, and 0509-MATSA. The five cloud images of the second row in Fig.3 respectively show 0511- MAWAR, 0514-NABI, 0516-VICENTE, 0518-DAMREY, and 0522-TEMBIN. The five cloud images of the first row in Fig.4 are respectively 0601-CHANCHU, 0603-EWINIAR, 0606-PRAPIROON, 0611-SONAMU, and 0613- SHANSHAN. The five cloud images of the second row in Fig.4 respectively show 0616-BEBINCA, 0617-RUMBIA，0619-CIMARON，0621-DURIAN, and 0623-Trami. 
Table 1. Scores of enhanced images with different PSNR (0425- NOCK-TEN)

\begin{tabular}{ccccccc}
\hline PSNR & HE & AFL & WYQ & XLZ & USM & ZCJ \\
\hline 2 & 150.36 & -328.45 & -263.02 & 195.06 & -1215.60 & $\mathbf{2 0 0 . 9 0}$ \\
4 & 168.49 & -268.79 & -212.06 & 194.99 & -1073.30 & $\mathbf{2 0 5 . 8 9}$ \\
6 & 180.84 & -196.56 & 100.21 & 194.89 & -953.32 & $\mathbf{2 1 0 . 9 9}$ \\
8 & 190.35 & 183.54 & 192.16 & 194.81 & -856.23 & $\mathbf{2 1 7 . 6 4}$ \\
10 & 197.57 & 213.16 & 209.92 & 194.74 & -784.43 & $\mathbf{2 2 3 . 5 2}$ \\
12 & 203.22 & 223.31 & 218.31 & 194.67 & -727.54 & $\mathbf{2 3 1 . 0 0}$ \\
14 & 207.19 & 228.17 & 223.27 & 194.61 & -688.84 & $\mathbf{2 3 5 . 2 1}$ \\
16 & 210.18 & 230.51 & 226.28 & 194.57 & -660.16 & $\mathbf{2 3 9 . 2 0}$ \\
18 & 212.10 & 231.77 & 228.37 & 194.53 & -643.19 & $\mathbf{2 4 1 . 9 5}$ \\
20 & 213.36 & 232.58 & 229.74 & 194.49 & -632.31 & $\mathbf{2 4 3 . 2 6}$ \\
\hline
\end{tabular}

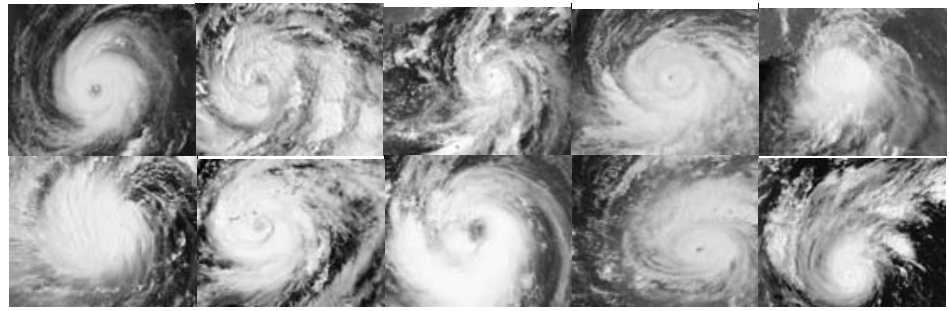

Fig. 2. Test typhoon cloud images (2004)

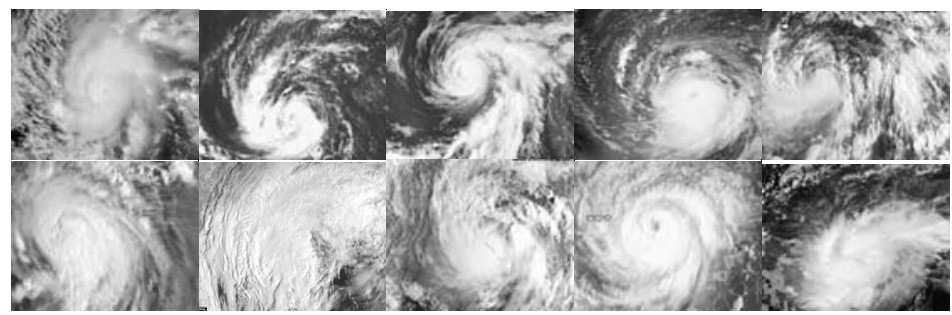

Fig. 3. Test typhoon cloud images (2005)

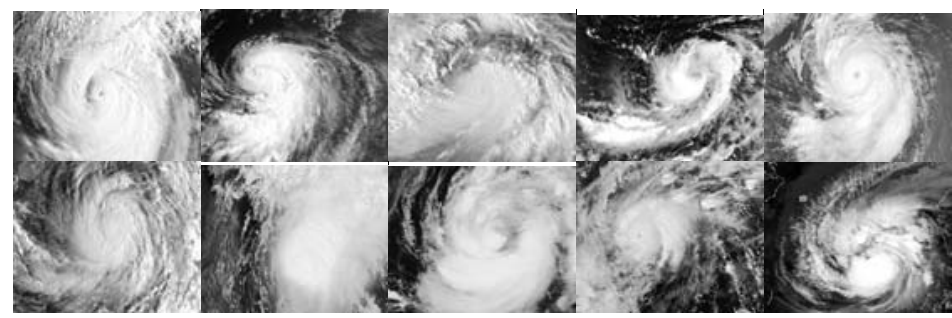

Fig. 4. Test typhoon cloud images (2006) 
In order to test the efficiency of the proposed measure, Table 2-Table 4 gives the scores of enhanced cloud images with different methods. Here all the PSNR of the test cloud images are set as $12 \mathrm{~dB}$. From Table 2-Table 4, we can see that the scores of ZCJ method is the highest compared with other five methods. Although some scores are higher than ZCJ method, for example, the two scores of AFL method and WYQ method are higher than ZCJ method in Table 3 and Table 4, the overall visual quality of the ZCJ method is the best. In a sum, the proposed assessment measure can be used to assess the visual quality of the enhanced typhoon cloud images. The visual quality is agreed with the objective index with the proposed assessment measure.

Table 2. Scores of enhanced images with different typhoon cloud images (2004)

\begin{tabular}{ccccccc}
\hline No. & HE & AFL & WYQ & XLZ & USM & ZCJ \\
\hline 0401 & 205.11 & 238.28 & 242.92 & 226.94 & -696.57 & $\mathbf{2 4 4 . 7 0}$ \\
0402 & 170.91 & 190.61 & 179.01 & 153.05 & -689.87 & $\mathbf{1 9 1 . 6 5}$ \\
0404 & 207.79 & 232.63 & 228.11 & 198.76 & -739.28 & $\mathbf{2 3 5 . 1 4}$ \\
0406 & 181.99 & 202.88 & 206.96 & 190.30 & -642.83 & $\mathbf{2 0 8 . 4 2}$ \\
0409 & 204.08 & 226.69 & 230.59 & 217.20 & -682.41 & $\mathbf{2 3 7 . 7 9}$ \\
0413 & 202.93 & 238.98 & 238.00 & 216.05 & -737.93 & $\mathbf{2 5 0 . 6 0}$ \\
0414 & 198.93 & 237.18 & 234.26 & 215.47 & -737.64 & $\mathbf{2 5 0 . 1 8}$ \\
0418 & 196.05 & 225.90 & 229.36 & 232.51 & -680.41 & $\mathbf{2 4 8 . 1 7}$ \\
0422 & 196.40 & 218.09 & 220.41 & 199.78 & -682.74 & $\mathbf{2 2 3 . 0 1}$ \\
0423 & 214.44 & 270.91 & 264.49 & 250.06 & -798.26 & $\mathbf{2 9 1 . 1 4}$ \\
\hline
\end{tabular}

Table 3. Scores of enhanced images with different typhoon cloud images (2005)

\begin{tabular}{ccccccc}
\hline No. & HE & AFL & WYQ & XLZ & USM & ZCJ \\
\hline 0502 & 137.73 & 166.98 & 165.33 & 131.92 & -654.88 & $\mathbf{1 6 7 . 1 3}$ \\
0503 & 202.15 & 251.57 & 244.09 & 214.70 & -774.18 & $\mathbf{2 5 9 . 2 9}$ \\
0504 & 212.19 & 248.39 & 248.37 & 228.13 & -753.06 & $\mathbf{2 6 5 . 2 6}$ \\
0505 & 208.90 & 237.52 & 239.85 & 217.80 & -724.94 & $\mathbf{2 4 5 . 6 6}$ \\
0509 & 191.77 & 209.57 & 168.76 & 158.38 & -762.65 & $\mathbf{2 1 5 . 8 3}$ \\
0511 & 178.79 & 181.53 & 185.12 & 164.04 & -636.87 & $\mathbf{1 8 4 . 6 4}$ \\
0514 & -123.29 & 144.11 & 137.94 & 114.88 & -627.51 & $\mathbf{1 4 4 . 1 5}$ \\
0516 & 174.07 & 184.54 & 166.53 & 140.15 & -711.63 & $\mathbf{1 8 3 . 3 8}$ \\
0518 & 166.57 & 180.96 & 177.49 & 161.89 & -656.87 & $\mathbf{1 8 3 . 1 1}$ \\
0522 & 219.32 & 268.87 & 269.63 & 252.78 & -769.90 & $\mathbf{2 8 8 . 1 4}$ \\
\hline
\end{tabular}


Table 4. Scores of enhanced images with different typhoon cloud images (2006)

\begin{tabular}{ccccccc}
\hline No. & HE & AFL & WYQ & XLZ & USM & ZCJ \\
\hline 0601 & 181.59 & 209.34 & 207.33 & 197.45 & -697.45 & $\mathbf{2 2 3 . 6 6}$ \\
0603 & 217.83 & 277.90 & 279.32 & 282.33 & -763.85 & $\mathbf{3 0 8 . 8 8}$ \\
0606 & -143.59 & 135.56 & 130.63 & 101.38 & -598.92 & $\mathbf{1 3 4 . 0 4}$ \\
0611 & 215.47 & 289.18 & 278.46 & 280.66 & -830.80 & $\mathbf{3 2 3 . 9 1}$ \\
0613 & 200.05 & 224.99 & 225.54 & 208.87 & -716.88 & $\mathbf{2 3 3 . 7 1}$ \\
0616 & 191.49 & 208.76 & 198.07 & 168.64 & -732.27 & $\mathbf{2 0 7 . 3 4}$ \\
0617 & 198.95 & 253.81 & 254.60 & 237.10 & -768.42 & $\mathbf{2 6 9 . 1 6}$ \\
0619 & 190.78 & 250.30 & 254.45 & 248.88 & -722.94 & $\mathbf{2 6 9 . 7 5}$ \\
0621 & 203.10 & 238.60 & 234.37 & 208.03 & -737.45 & $\mathbf{2 4 9 . 2 9}$ \\
0623 & 216.32 & 279.25 & 279.98 & 281.41 & -765.18 & $\mathbf{3 1 0 . 7 9}$ \\
\hline
\end{tabular}

\section{Conclusion}

This paper proposed an objective measure to assess the visual quality for typhoon cloud image enhancement. The measure can be used to evaluate the visual quality for satellite cloud image, especially for typhoon cloud image. It has a good assessment results by our designed experiments. This can provide accurate information for center and intensity prediction of the typhoon.

\section{Acknowledgments}

Part of the research is supported by the Grants for China Natural Science Foundation (40805048), China Zhejiang Province Natural Science Foundation (Y506203), Typhoon Research Foundation of Shanghai Typhoon Institute/China Meteorological Administration (2008ST01), Research foundation of State Key Laboratory of Severe Weather/Chinese Academy of Meteorological Sciences (2008LASW-B03). China Meteorological Administration, China National Satellite Meteorological Center is acknowledged for providing all the typhoon cloud images in this manuscript.

\section{References}

1. Albertz, J., Zelianeos, K.: Enhancement of satellite image data by data cumulation. Journal of Photogrammetry and Remote Sensing 45 (1990)

2. Fernandez-Maloigne, C.: Satellite images enhancement. In: Proceedings-International Symposium on Automotive Technology \& Automation, vol. 3, pp. 210-215 (1990)

3. Bekkhoucha, A., Smolarz, A.: Technique of images contrast enhancement: an application to satellite and aerial images. Automatique Productique Informatique Industrielle 26, 335-353 (1992) 
4. Karantzalos, K.G.: Combining anisotropic diffusion and alternating sequential filtering for satellite image enhancement and smoothing. In: Proceedings of SPIE - Image and Signal Processing for Remote Sensing IX, vol. 5238, pp. 461-468 (2004)

5. Wan, Y., Shi, D.: Joint Exact Histogram Specification and Image Enhancement Through the Wavelet Transform. IEEE Transactions on Image Processing 16, 2245-2250 (2007)

6. Temizel, A., Vlachos, T.: Wavelet domain image resolution enhancement. IEEE Proceedings Vision, Image and Signal Processing 153, 25-30 (2006)

7. Xiao, D., Ohya, J.: Contrast enhancement of color images based on wavelet transform and human visual system. In: Proceedings of the IASTED International Conference on Graphics and Visualization in Engineering, pp. 58-63 (2007)

8. Heric, D., Potocnik, B.: Image enhancement by using directional wavelet transform. Journal of Computing and Information Technology - CIT 14, 299-305 (2006)

9. Ercelebi, E., Koc, S.: Lifting-based wavelet domain adaptive Wiener filter for image enhancement. IEEE Proceedings Vision, Image and Signal Processing 153, 31-36 (2006)

10. Zeng, P., Dong, H., Chi, J., Xu, X.: An approach for wavelet based image enhancement. In: 2004 IEEE International Conference on Robotics and Biomimetics, pp. 574-577 (2004)

11. L. Gaoyong, O. David, H. Chris: Real-time wavelet denoising with edge enhancement for medical x-ray imaging. Proceedings of SPIE - The International Society for Optical Engineering, vol.6063, pp. 606303 (2006)

12. Belousov, A.A., Spitsyn, V.G., Sidorov, D.V.: Applying Wavelets and Evolutionary algorithms to automatic image enhancement. In: Proceedings of SPIE - The International Society for Optical Engineering, vol. 6522, pp. 652210 (2006)

13. Wang, Z., Bovik, A.C., Skith, H.R., Simoncelli, E.P.: Simoncelli: Image quality assessment: from error visibility to structural similarity. IEEE Transactions on Image Processing 13, 600-612 (2004)

14. Changjiang, Z., Xiaodong, W., Haoran, Z., Chunjiang, D.: An anti-noise algorithm for enhancing global and local contrast for infrared image. International Journal of Wavelet, Multi-resolution and Information Processing 5(1), 101-112 (2007)

15. Laine, A.F., Schuler, S., Fan, J., Huda, W.: Mammographic feature enhancement by multiscale analysis. IEEE Transactions on Medical Imaging 13(4), 725-752 (1994)

16. Ying-Qian, W., Pei-Jun, D., Peng-Fei, S.: Research on wavelet-based algorithm for image contrast enhancement. Wuhan University Journal of Natural Sciences 9, 46-50 (2004)

17. Zong, X., Laine, A.F.: De-noising and contrast enhancement via wavelet shrinkage and nonlinear adaptive gain. In: Published in wavelet applications III, proceedings of SPIE, vol. 2762, pp. 566-574 (1996) 